\title{
Altered Functions of Human Blood-Derived Vascular Endothelial Cells by Simulated Microgravity
}

\author{
Vidhya Ramaswamy ${ }^{1}$, Allison Goins ${ }^{1,2}$, and Josephine B. Allen ${ }^{1,2}$ \\ ${ }^{I}$ Department of Materials Science and Engineering, ${ }^{2}$ Institute of Cell and Tissue Science and Engineering, \\ University of Florida, Gainesville, FL
}

\begin{abstract}
Recently, the increase in incidence of cardiovascular degeneration associated with weightlessness has drawn much attention to the detrimental effects of space travel on cardiovascular health. Particularly, the regulatory role of the endothelium in cardiovascular degeneration has been studied extensively. The goal of this study was to understand the effects of simulated microgravity on the proliferative, secretory, and anti-thrombogenic functions of endothelial cells differentiated from human bloodderived progenitor cells. Exposure to simulated microgravity enhanced proliferation, as well as the release of soluble nitric oxide while downregulating the release of pro-inflammatory cytokines, such as interleukin-6 (IL-6). Interestingly, the cells also upregulated gene expression of heat shock protein 70 (hsp70), which may be a potential adaptation mechanism of the cells to altered gravity conditions. However, the secretory and proliferative functions
\end{abstract}

Key words: $\quad$ Microgravity; Endothelial

Progenitor Cells; Endothelial Cells; AntiThrombogenic

Correspondence to: Josephine Allen University of Florida

100 Rhines Hall

Gainesville, FL 32611

Telephone: 352-846-3328

E-mail: Jallen@mse.ufl.edu had no effect on the anti-thrombogenic functions of these cells. Their anti-coagulative and antithrombogenic abilities, as assessed by both upregulation of tissue plasminogen activator (tPA) and their ability to delay plasma clotting, were impaired on exposure to simulated microgravity. These results collectively provide a useful insight into various mechanisms involved in regulating anti-thrombogenic ability of the endothelium, as well as cardiovascular health in altered gravity conditions.

\section{INTRODUCTION}

Within the past 50 years of human spaceflight missions, several physiological threats to astronauts have been identified, including: losses in bone mineral density, muscle atrophy, shifts in the distribution of body fluids, and cardiovascular deconditioning (Yates and Kerman, 1998; Convertino, 2009; Versari et al., 2013). There has been a significant amount of research focused on studying the negative effects of cardiovascular degeneration and the development of possible strategies and countermeasures to address them (Mukai et al., 1994; Antonutto and di Prampero, 2003). Proper function of the cardiovascular system is largely dependent on the maintenance of healthy functioning vascular cells, namely endothelial cells (ECs) that line the vasculature and are in direct contact with circulating blood (Rubanyi, 1993; Douville et al., 1987). The ECs that line the vasculature form a thin layer called 


\section{Ramaswamy et al. -- Simulated Microgravity Alters Blood-Derived Endothelial Cell Functions}

the endothelium, and this layer of the vasculature plays a critical role in regulating blood flow, vascular permeability, and maintaining an actively anti-thrombogenic surface. The ability of the endothelium to perform these functions is largely governed by their highly-regulated secretory capabilities (Allen et al., 2010; Hristov et al., 2003). The endothelium has also been shown to respond to physiological mechanical cues—such as shear stress, cyclic stretch, and alterations to gravity-which cause them to undergo significant morphological and functional changes (Carlsson et al., 2003; Monici et al., 2015; Grimm et al., 2014; Gimbrone and Lelkes, 2003; Ali and Schumacker, 2002).

Supplementary to ECs, circulating progenitor cells (CPCs) also play a role in regulating the normal functioning of the vasculature. These CPCs reside in the bone marrow and are released into circulation upon stimulation (Urbich and Dimmeler, 2004; Allen et al., 2010; Zampetaki et al., 2008; Asahara et al., 1997). Once in circulation, they can integrate into the vasculature, differentiate into ECs, and participate in the repair of damaged vascular tissues. The long-term goal of this study is to fill the gap in knowledge regarding the effects of microgravity on the downstream function of CPCs after they have differentiated into ECs. These stem cells serve as a source of healthy vascular cells and play a very important role in the maintenance of vascular homeostasis (Kunz et al., 2006; Murasawa and Asahara, 2005; Yamamoto et al., 2003; Hristov et al., 2003). While the effect of weightlessness on mature ECs has been studied extensively, there is a dearth of information regarding the effect of microgravity on ECs derived from the circulating stem/progenitor cell population. Hence, it is important to study the effect of simulated microgravity on the functions of these cells to understand their implications during spaceflight and their possible role in the onset of cardiovascular dysfunction.

As a first step towards establishing an understanding of the effect of simulated microgravity on circulating progenitor-derived ECs, we modeled microgravity using a groundbased rotating wall vessel (RWV) bioreactor. The RWV was designed and developed at NASA's Johnson Space Center to simulate the effects of microgravity on cells in a ground-based culture system (Radtke and Herbst-Kralovetz, 2012; Martinez et al., 2015). The bioreactor set-up used in this study consisted of a cylindrical vessel/chamber that rotates around its horizontal axis with a coaxial tubular silicon membrane for gas exchange (Unsworth and Lelkes, 1998; Hammond and Hammond, 2001; Grimm et al., 2014; Klaus, 2001). The results obtained using simulated microgravity conditions utilizing the RWV have been confirmed and validated by experimental models in spaceflights (Cotrupi et al., 2005; Martinez et al., 2015; Versari et al., 2013; Versari et al., 2007).

This study reports the effect of simulated microgravity on the downstream functions of human blood-derived ECs, such as: proliferation, release of soluble factors nitric oxide (NO), interleukin-6 (IL-6), tissue plasminogen activator (tPA), gene expression of HSPA4 (gene encoding heat shock protein $70 \mathrm{kDa} \mathrm{A})$, and antithrombogenic potential. More specifically, this work is focused on the ability of these cells to adapt to hypergravity conditions and modulate these functions after short-term culture and expansion under simulated microgravity conditions. To our knowledge, this is the first report that provides valuable insight into the functionality of human blood progenitor cellderived ECs following exposure to simulated microgravity. This work provides a foundation for future work to elucidate vascular repair mechanisms taking place in the body in outer space.

\section{METHODS AND MATERIALS}

\section{Isolation of Peripheral Blood Mononuclear Cells from Human Blood}

About $45 \mathrm{ml}$ of circulating peripheral blood was collected from healthy donors by venipuncture in the presence of heparin (158 USP) anti-coagulant in vacutainer tubes (Thermo Fisher Scientific, Waltham, MA). For the purpose of clotting studies alone, blood was collected in vacutainer tubes containing Acid Citrate Dextrose (ACD) solution (Thermo Fisher Scientific) as the anti-coagulant. All blood collection procedures were performed in accordance with the rules and regulations of the Institutional Review Board at the University of Florida. Within $2 \mathrm{~h}$ of blood collection, the peripheral blood mononuclear cells 


\section{Ramaswamy et al. -- Simulated Microgravity Alters Blood-Derived Endothelial Cell Functions}

(PBMNCs) were isolated using density gradient centrifugation, as described in detail elsewhere (Allen et al., 2010; Allen et al., 2008). Approximately 80 million PBMNCs were obtained from the isolation process, then subsequently suspended in endothelial cell basal media-2 (EBM-2) (Lonza, Walkersville, MD) supplemented with $20 \%$ fetal bovine serum, human fibroblast growth factor (hFGF), vascular endothelial growth factor (VEGF), human epidermal growth factor (hEGF), human recombinant insulin-like growth factor (R3-IGF1), ascorbic acid, gentamicin, amphotericin, and heparin. Henceforth, this fully supplemented EBM-2 is referred to as endothelial cell growth media-2 (EGM-2).

\section{Culture and Differentiation of Endothelial Progenitor Cells}

The PBMNCs were plated at a density of 2$3 \times 10^{5}$ cells $/ \mathrm{cm}^{2}$ onto fibronectin-coated tissue culture polystyrene plates (BD Biosciences, Franklin Lakes, NJ) in complete cell culture media, as described above. The culture was maintained for 2-6 weeks and the cells were visually assessed to observe colony formation every day using an inverted phase-contrast microscope (Nikon TE2000U, Melville, NY). The first appearance of tightly-packed, cobblestone morphology, human endothelial-like (HE-like) cells in a given field of view was recorded as indicative of EC differentiation. The differentiated cells were sub-cultured and utilized within passages 1-3. Human umbilical vein endothelial cells (HUVECs) (Lonza) were used as a positive control for mature EC functionality. HUVECs were cultured in EGM-2 (with only 2\% FBS) and utilized within passages 3-5.

\section{Immunocytochemical Assessment of Differentiated Endothelial Cells}

The EC phenotype of the differentiated cells was confirmed using immunocytochemical analysis (herein after referred to as HE-like cells). Briefly, HE-like cells were fixed in $4 \%$ paraformaldehyde, and then blocked using 3\% bovine serum albumin for $1 \mathrm{~h}$. The cells were incubated overnight at $4^{\circ} \mathrm{C}$ with a primary antibody specific to von Willebrand factor (vWF) (diluted 1:100) (Abcam, Cambridge, UK) and CD31 (diluted 1:100) (Abcam). The cells were subsequently incubated with a fluorescein isothiocyanate tagged isotype-matched secondary antibody (Santa Cruz Biotechnology, Santa Cruz, $\mathrm{CA}$ ), and the nuclei were counter-stained using Hoechst 33342 (Thermo Fisher Scientific). Fluorescent images were obtained using an inverted fluorescent microscope (Nikon TE2000U, Melville, NY).

\section{Simulated Microgravity Culture of HE-Like Cells}

Cytodex 1 beads (Sigma Aldrich, St. Louis, MO) were swollen and sterilized using manufacturer's instructions (Cogoli, 1993; Hammond and Hammond, 2001; Radtke and Herbst-Kralovetz, 2012). The beads were coated with fibronectin (25 ng/ml) (Sigma Aldrich) to promote cell adhesion. HE-like cells obtained using the above mentioned procedure were seeded on the beads at a density of $3 \times 10^{6}$ cells per 250 mg of beads in ultra-low attachment cell culture plates. The culture was maintained for up to 4 days until complete confluence of cells on beads was obtained. The cell-coated beads were then transferred into the bioreactor chambers (Synthecon, Houston, TX), as shown in Figure 1A and Figure 1B, and cultured under simulated microgravity conditions. Briefly, the bioreactor was set to rotate at an initial speed of $8.5 \mathrm{rpm}$ to suspend the cells under simulated microgravity with minimum shear stress in a cell culture incubator with $5 \%$ carbon dioxide $\left(\mathrm{CO}_{2}\right)$. For all experiments, as a static control, the cell-coated beads were placed into the bioreactor chambers in a cell culture incubator; however, the chambers were not rotating and thus were maintained under normal gravity. Subsequently, the cultures were maintained for up to 6 days. About half of the cell culture media in the chamber was replaced every other day.

\section{Cell Proliferation Assessment}

Cell proliferation was assessed via changes in DNA concentration of HE-like cells cultured under both simulated microgravity and normal gravity over time. The double-stranded DNA (dsDNA) was quantified using Quant-it picogreen dsDNA assay kit (Thermo Fisher Scientific) following manufacturer instructions. Briefly, HElike cells on beads exposed to simulated microgravity were collected at 1, 2, 4, and 6 days 

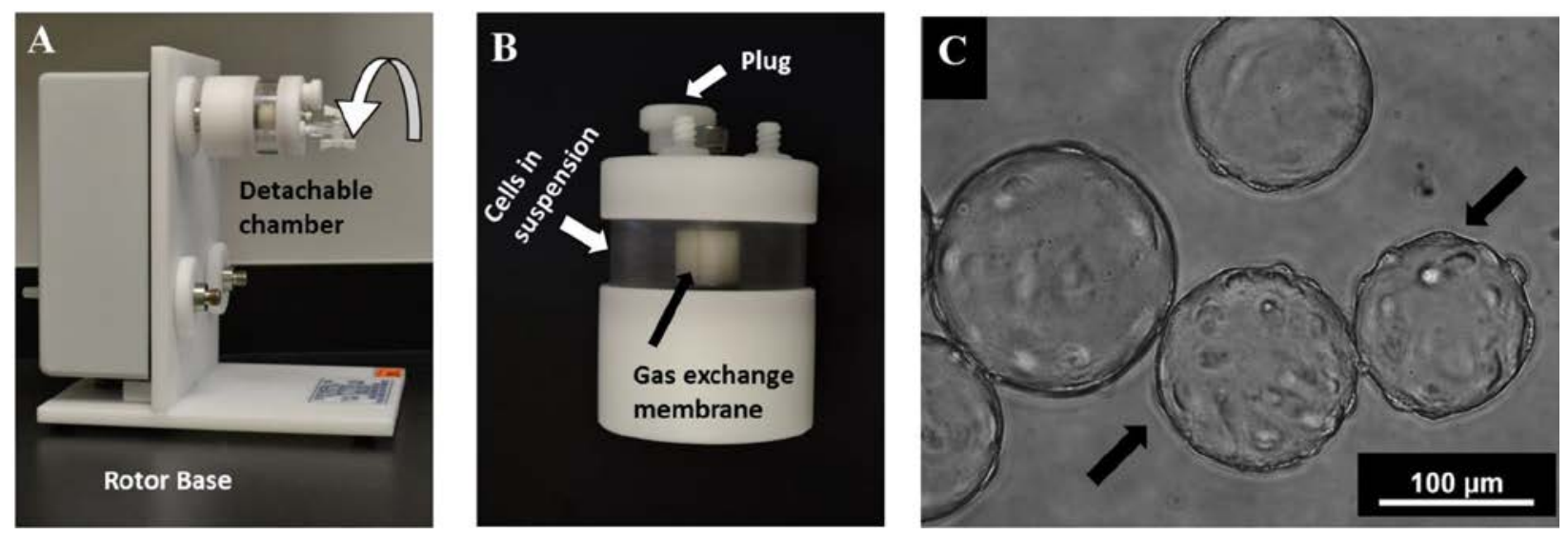

Figure 1. Bioreactor set-up and operation: (A) Synthecon RWV bioreactor set-up (B) RWV bioreactor detachable chamber (C) Representative 20X phase contrast image of HE-like cells cultured on cytodex-1 microcarrier beads. Scale bar is $100 \mu \mathrm{m}$. In panel C, the arrows indicate cell-covered beads.

of culture from the bioreactor chamber. Correspondingly, samples were collected from normal gravity controls at the same respective time points. The cells were then lysed using $0.1 \%$ triton-X to collect and quantify the intracellular DNA. The DNA concentrations of lysates were quantified and plotted versus time.

\section{Gene Expression}

To determine the effect of culture conditions on gene expression of hsp70 by the HE-like cells, quantitative reverse transcriptase polymerase chain reaction (qRT-PCR) was conducted. After 2 and 4 days of culture, total RNA was isolated from the HE-like cells cultured under normal and reduced gravity conditions using the RNeasy Mini Kit (Qiagen, Valencia, CA) according to manufacturer instructions. Subsequently, iScript cDNA Synthesis Kit (Bio Rad, Hercules, CA) was used to generate first-strand cDNA. The RNA sequences of human glyceraldehyde 3-phosphate dehydrogenase $(G A P D H)$ and heat shock protein 70 kDa A 4 (HSPA4) were identified and selected from the National Center for Biotechnology Information (NCBI) gene database, and corresponding primers for each of the genes were obtained from Integrated DNA Technologies (IDT, Coralville, IA). qRT-PCR was run on the Bio Rad CFX Connect (Bio Rad, Hercules, CA) using Maxima SYBR Green (Thermo Fisher Scientific, Waltham, MA) to quantify the expression of each target gene. Melt curve analysis was run to ensure amplification of non- specific PCR products did not affect the quantification results. Results for gene expression were normalized using $G A P D H$. The data obtained was derived from cells from a single donor population.

\section{Endothelial Cell Secretory Function}

The amount of NO, IL-6, and tPA secreted into the culture medium by the HE-like cells was assessed after 2 and 4 days of culture in both normal and simulated microgravity. Quantification was obtained utilizing the total NO assay kit (Cayman Chemical, Ann Arbor, MI), Human IL-6, and Human t-Plasminogen Activator/tPA Quantikine ELISA Kits ( $\mathrm{R}$ and D Systems, Minneapolis, MN), respectively, following manufacturer protocols. Briefly, the concentration of NO released into the medium was quantified based on the enzymatic conversion of nitrates to nitrites, and the photometric detection of total nitrite concentration via the Greiss reaction. The concentration of NO was normalized to cell density using the dsDNA concentration of the cells, and expressed in terms of nitrite concentration $(\mu \mathrm{M})$ per pg of dsDNA in order to draw comparisons between times in simulated microgravity.

\section{Plasma Clotting Kinetics}

The plasma clotting kinetics of the HE-like cells was assessed using a modified protocol previously reported (Allen et al., 2008; Ramaswamy et al., 2015). Briefly, human 
allogenic platelet-poor plasma was obtained via centrifugation from whole blood. HE-like cells on beads cultured for 2 and 4 days-both under simulated microgravity, as well as under normal gravity-were exposed to the $250 \mu$ of the plasma recalcified by the addition of $0.025 \mathrm{M}$ calcium chloride $\left(\mathrm{CaCl}_{2}\right)$. Following which, the absorbance at $405 \mathrm{~nm}$ was recorded using a Synergy H1 microplate reader (Biotek, Winooski, VT). As the plasma clots it becomes more opaque, thus an increase in absorbance with time is indicative of clot formation. HUVEC-coated microcarrier beads served as a positive control for anti-thrombogenic function of mature ECs. The full kinetic profile of clot formation, as well as the time taken to reach maximum absorbance, which is indicative of full clotting, was recorded and reported for each sample/control.

\section{Statistical Analysis}

All data are reported as mean $+/$ - standard deviations. A student 2-sample t-test was employed to compare two numerical means. Analysis of variance (ANOVA) with Tukey's multiple comparisons post hoc analysis was used to analyze and identify significant differences between 3 or more means. p-values $<0.05$ are reported as significant.

\section{RESULTS}

\section{Differentiation of Endothelial Progenitor Cells into Endothelial-Like Cells}

In this report, CPCs contained within the PBMNC fraction of whole blood were isolated then cultured in the presence of VEGF to induce differentiation along the endothelial lineage (Allen et al., 2010; Allen et al., 2008; Motlagh et al., 2007; Ramaswamy et al., 2015). Differentiation into endothelial phenotype was confirmed by the formation of colonies with EC specific cobblestone morphology, as shown in Figure 2A. Colony formation was observed between 2-6 weeks of culture. In all cases, the observation for colonies was concluded after 50 days. The process was repeated for three populations, each from a different donor. The data reported are representative of these three different populations. The endothelial phenotype of these cells were confirmed and validated by the maintenance of the characteristic cobblestone morphology in subsequent culture, as shown in Figure 2B, and positive immunocytochemical staining for CD31, as shown in Figure 2C, and vWF, as shown in Figure 2D-both of which are specific for EC phenotype.

\section{Cell Proliferation}

The effect of simulated microgravity $(\mu g)$ was assessed for differentiated HE-like cells and compared to the kinetics of proliferation under normal gravity (ng) conditions. The proliferative capacity of the HE-like cells was assessed by quantifying the change in DNA over 1, 2, 4, and 6 days of culture. The results indicated the concentration of DNA in the normal gravity (ng) cultured cells was initially higher than the simulated microgravity $(\mu g)$ up to 2 days of culture $(\mathrm{p}<0.05)$, as shown in Figure 3 [dsDNA concentration $(\mu \mathrm{g} / \mathrm{ml})$ after 1 day: ng 0.098 +/0.002, $\mu g$ 0.073+/- $0.013 \mathrm{p}<0.05$; after 2 days: $\mathrm{ng}$ $0.110+/-0.005, \quad \mu g \quad 0.093+/-0.007 \quad \mathrm{p}<0.05]$. However, the cells cultured under simulated microgravity caught up by day 4 and showed no significant difference in DNA content [dsDNA concentration $(\mu \mathrm{g} / \mathrm{ml})$ after 4 days: $\mathrm{ng} 0.116+/-$ $0.005 ; \mu g 0.113+/-0.003$ not significant]. After 4 days, the HE-like cells under simulated microgravity increased their rate of proliferation, as indicated by a higher concentration of DNA, when compared to the normal gravity control at day 6 [dsDNA concentration $(\mu \mathrm{g} / \mathrm{ml})$ after 6 days: ng 0.151+/- 0.010, $\mu g$ 0.173+/- 0.003 p<0.05].

\section{Gene Expression of hsp70}

Furthermore, we investigated the ability of HE-like cells to sense simulated microgravity as a stressful condition and adapt to it by regulating the levels of genes encoding stress proteins, such as hsp70. Our results showed that simulated microgravity cultured cells upregulated gene expression of HSPA4-which belongs to the family of genes encoding hsp70 protein-after 2 days of culture, as shown in Figure 4 [after 2 days: $n g$ 0.496+/- 0.129, $\mu g$ 1.0+/- $0.306 \mathrm{p}<0.05]$. However, after 4 days in culture, there is no statistical difference in the level of HSPA4 between simulated microgravity and normal gravity [after 4 days: $\mathrm{ng} 0.346+/-0.154 ; \mu g$ $0.726+/-0.101$ not significant]. The data generated was from a representative population of HE-like cells isolated from a single donor. 

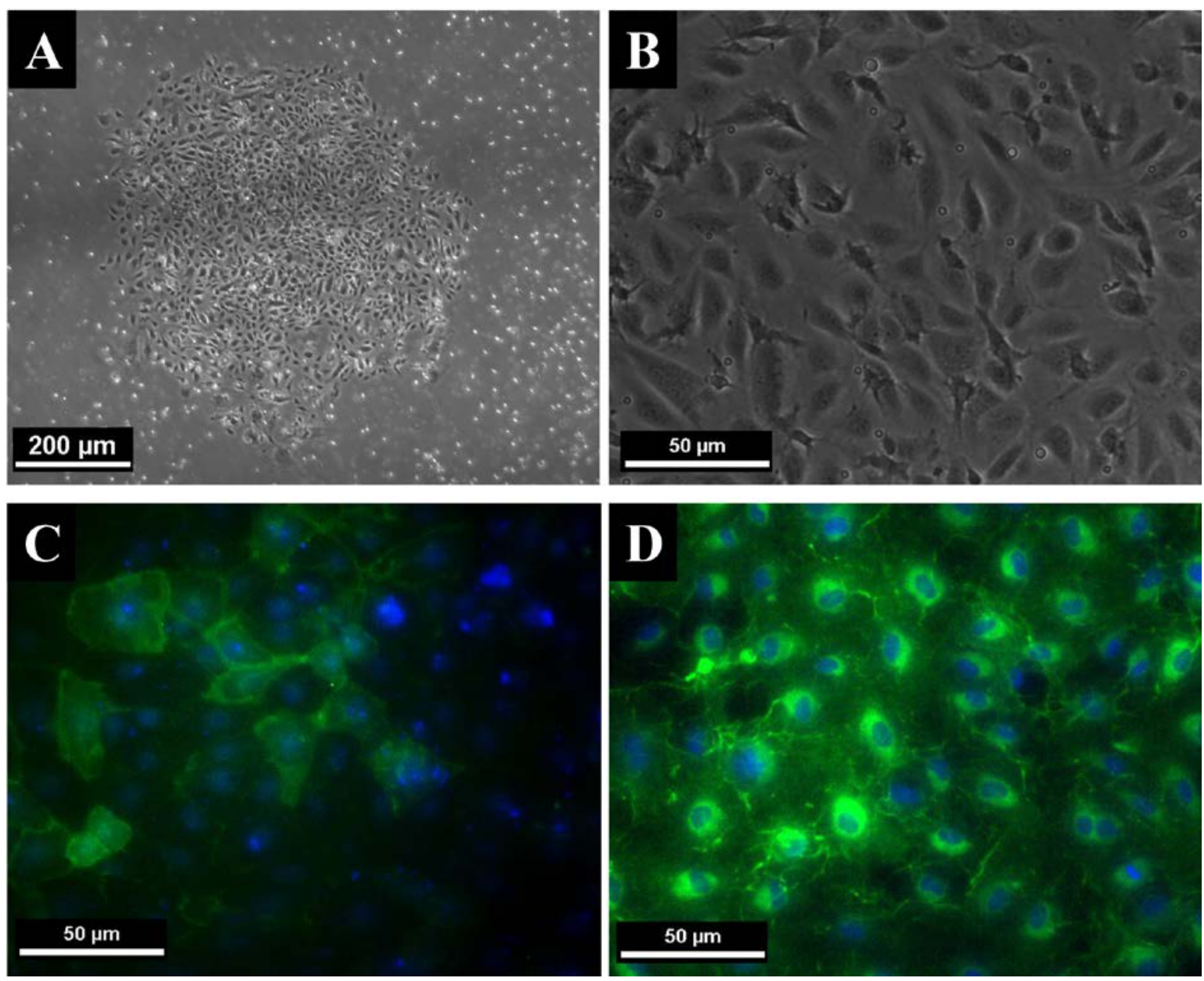

Figure 2. Isolation of PBMNCs and differentiation into ECs: (A) Representative phase contrast image of PBMNC-derived colony formation of HE-like cells; Scale bar $200 \mu \mathrm{m}$ (B) Representative phase contrast image of characteristic endothelial cobblestone morphology of HE-like cells in subsequent culture (20X). Representative 20X fluorescent images of positive immunocytochemical staining of HE-like cells for (C) CD31 and (D) von Willebrand factor, both shown in green; nuclei were counterstained with Hoechst 33342, shown in blue. Panel B - Panel D scale bar is 50 rm.

\section{Endothelial Cell Secretory Functions}

\section{Nitric oxide}

Results indicate that HE-like cells cultured under simulated microgravity for 2 days released higher amounts of soluble NO when compared to the respective normal gravity controls [NO concentration ( $\mu \mathrm{M} / \mathrm{pg}$ of dsDNA): ng $0.64+/-$ 0.308, $\mu g$ 1.77+/- $0.202 \mathrm{p}<0.05]$. However, after 4 days of culture the level of NO released was statistically similar between both groups [NO concentration $(\mu \mathrm{M} / \mathrm{pg}$ of dsDNA): $n g 2.49+/-$ $0.050 ; \mu g 2.51+/-0.079$ not significant], as shown in Figure 5A.

\section{Interleukin-6}

Assessment of the release of soluble IL-6, a major pro-inflammatory cytokine, show that culture under simulated microgravity conditions for 2 and 4 days led to significant decrease in secretion of soluble IL-6 in the cell culture medium when compared to normal gravity cultured controls [IL-6 concentration $(\mathrm{pg} / \mathrm{ml})$ after 2 days: ng 46.3+/-1.0, $\mu g$ 11.71+/-0.88 $\mathrm{p}<0.05$; after 4 days: ng $97.013+/-0.592$; $\mu g 53.697$ +/$9.196 \mathrm{p}<0.05]$, as shown in Figure 5B. 


\section{Ramaswamy et al. -- Simulated Microgravity Alters Blood-Derived Endothelial Cell Functions}

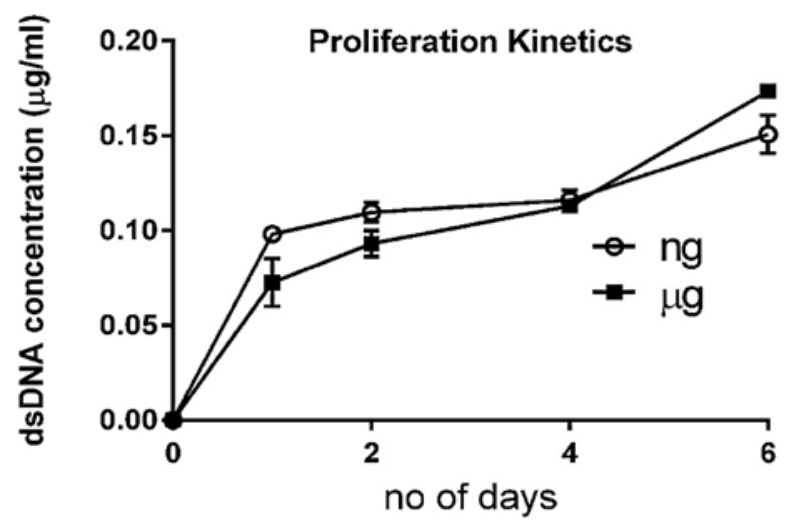

Figure 3. Proliferation kinetics: Representative proliferation kinetics curve HE-like cells cultured under microgravity $(\mu g)$ vs. cells maintained in normal gravity (ng), as measured by DNA concentration in cell lysates. Data shown are Mean +/- SD; $\mathbf{n}=3$.

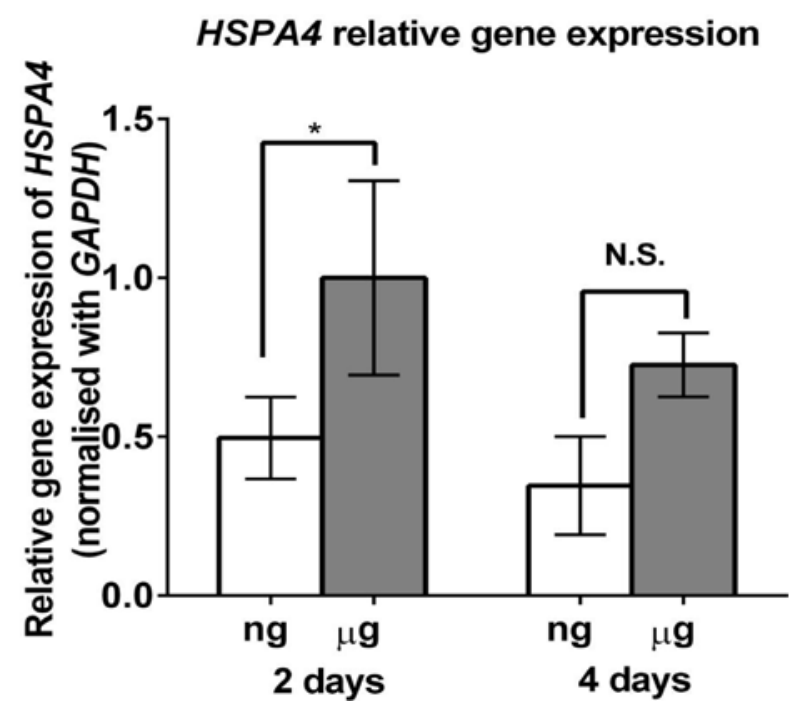

Figure 4. Gene expression: Representative relative gene expression of HSPA4 by microgravity $(\mu g)$ cultured HE-like cells vs. cells maintained in normal gravity (ng) over 2 and 4 days of culture. Data shown are Mean +/- SD; $n=3$. N.S. indicates no statistically significant difference $(p>0.05)$ and * indicates a statistically significant increase $(p<0.05)$.

\section{Tissue plasminogen activator}

Quantification of the release of soluble tPA into the surrounding cell culture medium showed that tPA released by HE-like cells cultured under simulated microgravity conditions for 2 and 4 days were significantly lower than their respective normal gravity cultured controls [tPA concentration (ng/ml) after 2 days, ng 4.572+/-
0.370, $\mu g$ 2.01+/- $0.263 \mathrm{p}<0.05$; after 4 days: $n g$ $13.26+/-0.557$; $\mu g 9.867+/-0.528 \mathrm{p}<0.05$ ], as shown in Figure 5C.

\section{Plasma Clotting Kinetics}

To further study and confirm the direct impact of simulated microgravity on the ability of these cells to regulate clotting, a modified version of plasma clotting assay was performed. This assay provided a robust and direct method to assess and compare the anti-thrombogenic potential of HElike cells cultured under different gravity conditions. Once clotting was activated, progress to full clot formation was assessed spectrophotometrically by continuous monitoring of the change in absorbance over $30 \mathrm{~min}$. The clotting profiles of recalcified plasma incubated with HE-like cells cultured in both simulated microgravity, as well as normal gravity, for both 2 and 4 days, is shown in Figure 6. HE-like cells cultured in normal gravity exhibited a significant delay in the onset of clotting, as indicated by the rightward shift in the kinetic profile, indicating a degree of anti-thrombogenic function. This was in contrast to the kinetic profile of plasma clotting when incubated with HE-like cells cultured under simulated microgravity, which show an earlier onset to clot formation. These trends are seen at both 2 and 4 days of culture, as shown in Figure $6 \mathrm{~A}$ and Figure 6B. As a control, $\mathrm{CaCl}_{2}$ was omitted from a subset of samples and the plasma did not clot (data not shown). We also quantified and reported the time taken to reach maximum absorbance, indicating complete clotting of plasma on the cell-covered beads. Our results indicate that recalcified plasma clots faster when incubated with HE-like cells cultured under simulated microgravity, as compared to the longer time it takes plasma to clot when incubated with HE-like cells cultured under normal gravity, as shown in Figure 6C [clotting time (min): with 2 day cultured HE-like cells: ng 14.31+/- 2.199, $\mu g$ $10.001+/-1.395 \mathrm{p}<0.05$; with 4 day cultured HElike cells: ng 16.569 +/- 1.357; $\mu g$ 12.456 +/$1.457 \mathrm{p}<0.05]$. HUVEC-coated beads were utilized as the control population of mature ECs and showed the most delayed plasma clotting (19.3 +/- 1.9 min) (data not shown). Similarly, naked beads devoid of HE-like cells were also assessed and showed no delay in plasma clot formation (data not shown). 
A

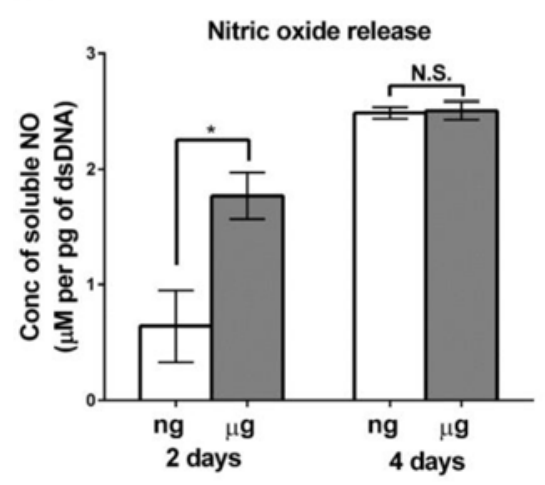

B

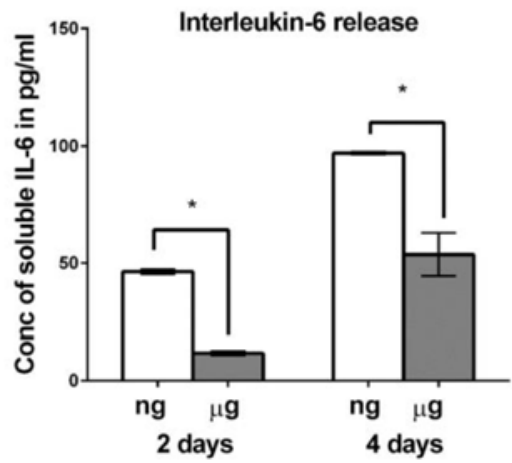

C

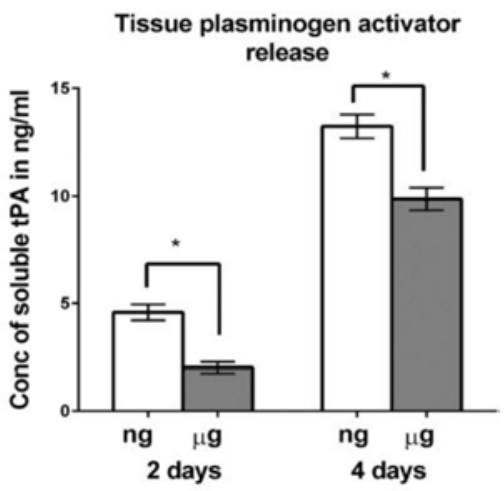

Figure 5. Secretory functions of cells: Representative results of quantification of release of (A) Nitric oxide and (B) Interleukin-6 by microgravity $(\mu \mathrm{g})$ cultured HE-like cells vs. cells maintained in normal gravity (ng) over 2 and 4 days of culture. Data shown are Mean +/- SD; $n=3$. N.S. indicates no statistically significant difference $(p>0.05)$ and $*$ indicates a statistically significant increase $(p<0.05)$.

A

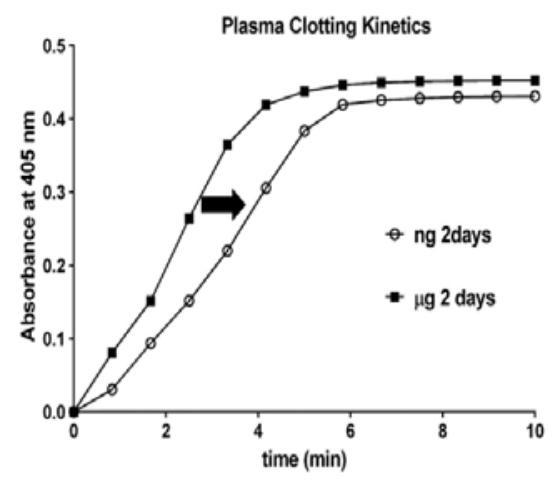

B

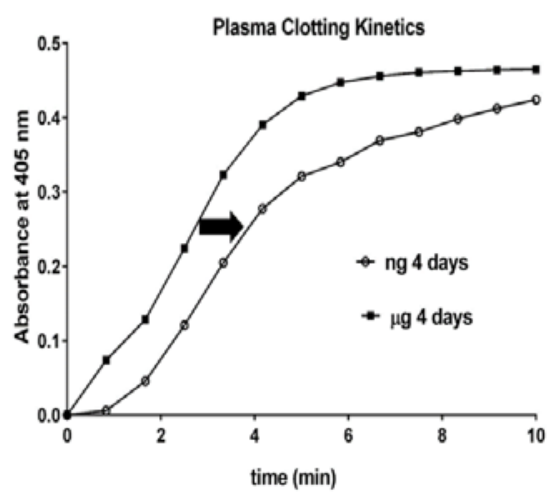

$\mathrm{C}$

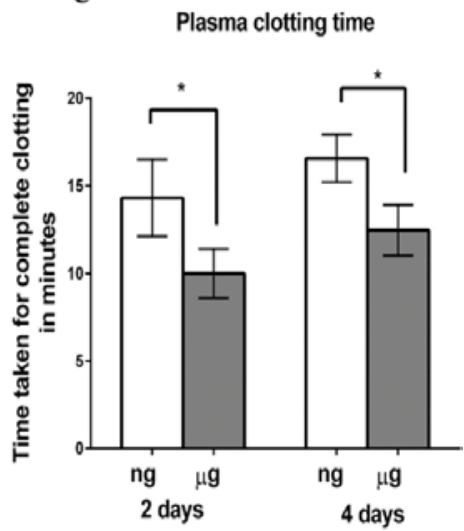

Figure 6. Plasma clotting kinetics: Recalcified plasma clotting kinetics for human plasma incubated with HElike cells cultured in normal vs. simulated microgravity for (A) 2 days and (B) 4 days. Data show absorbance plotted vs. time. Arrows indicate the rightward shift in the normal gravity conditions, indicative of delayed clotting. SD error bars have been omitted for visual clarity. Data shown are Means; $n=3$. (C) Representative result showing quantification of the time taken for recalcified plasma to clot (in minutes) when incubated with HE-like cells cultured for 2 and 4 days in normal vs. simulated microgravity. Data shown are Mean +/SD; $\mathbf{n}=6$. * indicates a statistically significant increase $(\mathbf{p}<0.05)$.

\section{DISCUSSION}

The physiological threats faced by astronauts as a direct consequence of human spaceflight have been identified and studied extensively in the recent past (Yates and Kerman, 1998; Versari et al., 2013; Wehland et al., 2013). The cardiovascular system of astronauts has been known to be affected by orbital spaceflight with the changes manifesting as cardiovascular deconditioning (Mukai et al., 1994; Versari et al., 2013; Convertino, 2009). From a clinical perspective, deconditioning is observed as a: (1) decrease of circulating blood and interstitial fluid volumes, (2) decrease of arterial blood diastolic pressure, (3) decrease of ventricular stroke volume, (4) decrease of estimated left ventricular mass, and (5) a resetting of the carotid baroreceptors. The deconditioning effects of 
microgravity manifest mostly upon the reentry to Earth (Antonutto and di Prampero, 2003). Maintenance of vascular homeostasis lies in the proper function of the vascular cells-specifically, the ECs that line the vessel wall. This work is focused on understanding the effects of short-term simulated microgravity on the downstream functions of ECs derived from whole blood. Previous work has shown the exposure of mature ECs to simulated microgravity (for as little as a few minutes to a few days) results in structural and morphological changes, as well as modifications to both gene and protein expression (Zhang et al., 2010; Uva et al., 2002; Grenon et al., 2013; Versari et al., 2007; Monici et al., 2015; Cogoli, 1993; Carlsson et al., 2003). These results have been observed and validated in both spaceflight experiments, as well as ground-based microgravity simulators, which was used here in our experiment. However, there is very limited literature on the effect of altered gravity conditions on blood-derived circulating endothelial progenitor cell (EPC) derived ECs. EPCs are a small population of progenitor cells that reside in bone marrow and, upon stimulation, are released into circulating peripheral blood. EPCs, along with the endothelium, are critical to the maintenance of vascular homeostasis, which includes regulating the hemodynamics of blood flow, vessel wall tone, and the secretion of important signaling molecules (Murasawa and Asahara, 2005; Zampetaki et al., 2008; Allen et al., 2010). Previous studies have shown the ability of circulating progenitor derived ECs to function in a similar manner to mature ECs, including their ability to regulate clotting mechanisms in vitro (van Hinsbergh, 2012; Ramaswamy et al., 2015; Stern et al., 1991; Allen et al., 2010; Allen et al., 2008). Despite the conflicting reports on the suitability of using EPCs for the study of cardiovascular diseases (Balistreri et al., 2015), this data shows the promising potential of EPCs to act as a repair cell population in cardiovascular regeneration. Therefore, this work is an important stepping stone towards understanding the role played by EPCs for the onset of cardiovascular deconditioning during space travel, as well as developing possible cell-based therapies. As a foundation for our work, our group has previously shown the effect of simulated microgravity on the differentiation and downstream anti-thrombogenic functions of porcine EPCs (Ramaswamy et al., 2015).

In this study, CPCs contained within the PBMNC fraction of whole blood were differentiated into cells with endothelial phenotype, termed HE-like cells. We further demonstrated that the HE-like cells expressed mature EC markers, vWF and CD31, and maintained the characteristic cobblestone morphology. To simulate microgravity culture conditions, HE-like cells were seeded onto fibronectin-coated microcarrier beads. A RWV bioreactor system-designed originally by NASA - was used to simulate a reduction in timeaveraged gravitational vector acting on these cells, reducing it to about $10^{-2}$ g. For simplicity, this reduced gravity condition is referred to as microgravity (Cotrupi et al., 2005; Villa et al., 2005; Carlsson et al., 2003). Although RWV systems do not mimic actual microgravity as experienced by astronauts, they are a useful tool to provide insight into the behavior of various cell systems in space (Hammond and Hammond, 2001; Radtke and Herbst-Kralovetz, 2012). The culture was maintained by adjusting the rotational speed just enough to maintain the vessel wall and the medium containing cells bound to microcarrier beads rotating at the same speed.

One of the primary and most important functions of the HE-like cells is their ability to adapt to changing conditions and to continually proliferate. Our results showed an initial decrease in cell growth, which was reversed over increased time in culture. A similar trend in proliferation has been shown for a mature EC population upon exposure to simulated microgravity (Carlsson et al., 2002; Versari et al., 2007). This data indicates that HE-like cells function similar to mature EC populations when exposed to simulated microgravity, thus enhancing their ability to proliferate. Additionally, this trend also indicates a potential recovery effect in the proliferative capacity of these ECs and HE-like cells. Such a proliferation effect has been reported in PBMNCs (Ritz et al., 2006) and other mature EC populations (Cotrupi and Maier, 2004; Villa et al., 2005; Versari et al., 2007; Carlsson et al., 2003). This trend also indicates the potential ability of these ECs to sense the change in gravitational conditions as stress, as well as their ability to recover from this stress, by enhancing 


\section{Ramaswamy et al. -- Simulated Microgravity Alters Blood-Derived Endothelial Cell Functions}

proliferation and cell growth (Cotrupi and Maier, 2004).

To further investigate the adaptive capacity of the ECs when exposed to changes in gravitational conditions, we quantified the regulation of gene expression of HSPA4. The HSPA4 gene belongs to the family of heat shock protein 70 . Heat shock proteins (hsp) have been known to play an important role in cell protection and repair upon stress, as well as under certain non-stressful conditions (Cotrupi and Maier, 2004; Kiang, 1998; Beere and Green, 2001). More specifically, hsp70 has been identified as a molecular chaperone which can inhibit apoptosis. Mechanistically, hsp70 inhibits apoptosis in cells by preventing the constitution of the apoptosome and by protecting against the apoptogenic effects of apoptosis-inducing factors (AIF) targeted to the extramitochondrial compartment (Ravagnan et al., 2001; Beere and Green, 2001). Previous studies have shown the ability of mature EC populations to sense simulated microgravity as a stressful condition and upregulate proteins, such as hsp70, and maintain their ability to proliferate (Cotrupi et al., 2005; Cotrupi and Maier, 2004; Carlsson et al., 2003; Carlsson et al., 2002). Consistent with this trend, our results indicated that culture under simulated microgravity conditions led to an initial upregulation of gene expression of HSPA4 over 2 days of culture, but by day 4 the levels were similar between the normal and simulated microgravity conditions. This data provides mechanistic insight into the ability of the HE-like cells to survive and proliferate under "stressful" conditions, such as simulated microgravity, by regulating stress proteins, such as hsp70.

Another important molecule that regulates EC function is NO. NO is an important regulator of various important functions of the endothelium, such as cell proliferation, angiogenesis, differentiation, and anti-thrombogenicity (Gamboa et al., 2013; van Hinsbergh, 2012). Our results showed an initial increase in the release of NO by the HE-like cells when cultured under short-term simulated microgravity conditions. Previous studies have reported a simulated microgravity-mediated increase in endothelial nitric oxide synthase (eNOS) protein levels, as well increased NO production at 48 hours and 72 hours of simulated microgravity exposure, respectively, by mature ECs (Cotrupi et al., 2005;
Grenon et al., 2013). Also, a study by Spisni et al. (2006, 2003) reported no change in eNOS or inducible nitric oxide synthase (iNOS) protein levels, yet an increase in NO production at 24 hours of simulated microgravity exposure. This increased level of NO may also indicate another possible recovery mechanism of the cells to adapt to the changing gravitational forces (Vaziri et al., 2000; Versari et al., 2007; Cotrupi and Maier, 2004; Carlsson et al., 2002). More importantly, an increase in expression of $\mathrm{NO}-\mathrm{a}$ known vasodilator-leads to increased relaxation and lowering of blood pressure, which is known to disrupt sympathetic flow, thus leading to orthostatic intolerance (Gamboa et al., 2013; Vaziri et al., 2000). Hence, this may also provide a possible insight into cardiac dysfunction caused by space travel.

The relationship between endothelial dysfunction and inflammatory response leading to cardiovascular complications, such as atherosclerosis, has been widely studied in the recent past (Trepels et al., 2009; Piro et al., 2005; van Hinsbergh, 2012). Immune dysfunction in astronauts in the space environment has also been studied extensively (Boonyaratanakornkit et al., 2005; Hughes-Fulford, 2011; Cogoli, 1993). A potential anti-inflammatory response to short-term culture under simulated microgravity by the downregulation of pro-inflammatory cytokines has been reported by multiple studies (Grenon et al., 2013; Cotrupi et al., 2005). These findings have also been implicated to be mediated by changes in cytoskeletal arrangement in simulated microgravity (Grenon et al., 2013). Consistent with the literature reports for mature ECs, our results showed a decrease in expression of IL-6, an important pro-inflammatory and antiproliferative cytokine (May et al., 1989). It is noteworthy that the inverse relationship between NO and IL-6 that was seen in this study has previously been reported for ECs (Deakin et al., 1995; Orshal and Khalil, 2004). Hence, the downregulation of IL-6 may play an important role in the response of the HE-like cells to simulated microgravity since low amounts of IL-6 might contribute to cell growth, as well as to the enhancement of NO synthesis. Similarly, the ability of hsp70 to inhibit the synthesis of proinflammatory cytokines in ECs has also been reported (Borges et al., 2012; Jacquier-Sarlin et 


\section{Ramaswamy et al. -- Simulated Microgravity Alters Blood-Derived Endothelial Cell Functions}

al., 1994; Luo et al., 2008). This altered immune function observed for the HE-like cells not only has implications in their regulatory role regarding the overall immune response of the body during space travel, but also additional implications on the effect of space travel on overall cardiovascular health.

The anti-thrombogenic behavior of ECs is important to maintain a healthy vascular system and to prevent cardiovascular complications such as thrombosis (Rubanyi, 1993). Healthy endothelium secretes various factors that are important in regulating coagulation and fibrinolysis (Stern et al., 1991; van Hinsbergh, 2012). Tissue plasminogen activator (tPA), an important protein released by ECs, has been shown to be a main factor in the activation of the fibrinolytic system, which is involved in dissolution of clots. Specifically, tPA activates the conversion of clot-bound plasminogen to plasmin, which leads to clot lysis (Van Guilder et al., 2005; Levin et al., 1984). An increase in expression of tPA has been directly correlated with increased bleeding, while a low expression of tPA has been implicated in thrombosis (Maiello et al., 1992). By assessing the expression of tPA released by HE-like cells, our data indicates simulated microgravity mediated an increase in expression of tPA over 2 and 4 days of culture. This indicates that exposure of HE-like cells to simulated microgravity can potentially decrease the antithrombogenic potential of the cells. In order to more directly study the anti-coagulative functions of the HE-like cells, we studied the ability of these cells to delay clotting of human blood-derived allogenic plasma. The clotting data corroborated the assessment of tPA secretion, in that HE-like cells exposed to simulated microgravity were not able to delay clot formation, as well as the normal gravity cultured controls over 2 and 4 days of culture. We observed that both populations, normal and simulated microgravity, show antithrombogenic function; however, this function was hindered with exposure to simulated microgravity. This data collectively shows that exposure to simulated microgravity negatively influences the anti-thrombogenic function of HElike cells relative to those cultured under normal gravity conditions. This inhibition of antithrombogenic functionality of the cells potentially provides insight into thrombosis and other coagulation processes in the body which have been known to directly cause cardiovascular deconditioning. Although the ability of simulated microgravity to directly affect platelet functions has been shown before (Dai et al., 2009), to our knowledge the effect of simulated microgravity on direct anti-thrombogenic properties of EPCderived ECs, such as inhibition of plasma clotting, has not been reported thus far.

It is important to note that decreased production of inflammatory cytokines such as IL6 , as well as increased production of NO, have been known to synergistically inhibit clotting processes in the body (Gamboa et al., 2013; Allen et al., 2008; van Hinsbergh, 2012; Trepels et al., 2009). However, in this study, we observed that although there was a significant reduction in the release of IL-6 and an increase in release of NO under simulated microgravity, the cells were able to induce faster clotting of plasma. It is also interesting to note that although culture under simulated microgravity conditions affects various secretory and proliferative mechanisms of the HElike cells, these functions seem to not have an effect on the downstream ability of the cells to delay clotting. This indicates the need to study extensively the effect of simulated microgravity on various alternate and synergistic mechanisms involved in both inflammatory, as well as thrombogenic processes. Also, the relatively small sample size ( $\mathrm{n}=3$ donor EPC populations) limits the detection of small statistical differences between samples. Nevertheless, this report provides a useful insight into the modulation of clotting mechanisms, as well as secretory and proliferative ability of blood-derived ECs when exposed to reduced gravity conditions. Since these cells are in direct contact with circulating blood, this study will prove to be particularly important when assessing their role in cardiovascular deconditioning during space travel, as well as to develop countermeasures to ameliorate or possibly reverse this effect.

\section{CONCLUSIONS}

The results of the present study demonstrated that the populations of human blood EPC-derived ECs when exposed to simulated microgravity proliferate faster, release higher amounts of soluble NO, and lower amounts of pro- 
inflammatory cytokines, such as IL-6, when compared to their normal gravity cultured controls. This can possibly be attributed to the ability of the cells to sense simulated microgravity as a "stressful" condition and adapt by upregulating gene expression of hsp70. However, these secretory, as well as proliferative functions had no effect on the anti-thrombogenic functions of these cells. Their anti-coagulative and antithrombogenic ability as assessed by both upregulation of tPA, as well as their ability to delay plasma clotting, were impaired upon exposure to simulated microgravity. This study is the first step towards understanding the ability of these repair cells to regulate clotting mechanisms and cardiovascular health in space. Further studies to assess alternate and synergistic mechanisms involved in regulation of cardiovascular health, as well as inflammatory dysfunction under microgravity, are required to develop possible cell-based countermeasures to prevent cardiovascular deconditioning due to space travel.

\section{ACKNOWLEDGEMENTS}

This work is funded by National Aeronautics and Space Administration, Space Biology Division, with Grant \# NNX13AM03G to J. Allen.

\section{AUTHOR DISCLOSURE STATEMENT}

No competing financial interests exist.

\section{REFERENCES}

Ali MH, Schumacker PT (2002) Endothelial responses to mechanical stress: where is the mechanosensor? Critical Care Medicine 30(5 Suppl): S198-S206

Allen JB, Khan S, Lapidos KA, Ameer GA (2010) Toward engineering a human neoendothelium with circulating progenitor cells. Stem Cells 28(2): 318-328

Allen JB, Khan S, Serrano MC, Ameer GA (2008) Characterization of porcine circulating progenitor cells: toward a functional endothelium. Tissue Engineering Part A 14(1): 183-194

$\begin{array}{ccr}\text { Antonutto G, di } & \text { Prampero PE } & \text { (2003) } \\ \text { Cardiovascular } & \text { deconditioning } & \text { in } \\ \text { microgravity: } & \text { some possible } & \text { counter- }\end{array}$ measures. European Journal of Applied Physiology 90(3-4): 283-291

Asahara T, Murohara T, Sullivan A, Silver M, van der Zee R, Li T, Isner JM (1997) Isolation of putative progenitor endothelial cells for angiogenesis. Science 275(5302): 964-967

Balistreri CR, Buffa S, Pisano C, Lio D, Ruvolo G, Mazzesi G (2015) Are endothelial progenitor cells the real solution for cardiovascular diseases? Focus on controversies and perspectives. BioMed Research International, 2015: 835934

Beere HM, Green DR (2001) Stress management heat shock protein-70 and the regulation of apoptosis. Trends in Cell Biology 11(1): 6-10

Boonyaratanakornkit JB, Cogoli A, Li CF, Schopper T, Pippia P, Galleri G, Meloni MA, Hughes-Fulford M (2005) Key gravitysensitive signaling pathways drive $\mathrm{T}$ cell activation. Federation of American Societies for Experimental Biology (FASEB) Journal 19(14): 2020-2022

Borges TJ, Wieten L, van Herwijnen MJC, Broere F, van der Zee R, Bonorino C, van Eden W (2012) The anti-inflammatory mechanisms of hsp70. Frontiers in Immunology 3: 95

Carlsson SIM, Bertilaccio MTS, Ascari I, Bradamante S, Maier JAM (2002) Modulation of human endothelial cell behavior in simulated microgravity. Journal of Gravitational Physiology 9(1): P273-P274

Carlsson SIM, Bertilaccio MTS, Ballabio E, Maier JAM (2003) Endothelial stress by gravitational unloading: effects on cell growth and cytoskeletal organization. Biochimica et Biophysica Acta (BBA) Molecular Cell Research 1642(3): 173-179

Cogoli A (1993) The effect of hypogravity and hypergravity on cells of the immune system. Journal of Leukocyte Biology 54(3): 259-268

Convertino VA (2009) Status of cardiovascular issues related to spaceflight: implications for future research directions. Respiratory Physiology \& Neurobiology 169(Suppl): S34-S37

Cotrupi S, Maier JAM (2004) Is HSP70 upregulation crucial for cellular proliferative response in simulated microgravity? Journal of Gravitational Physiology 11(2): P173-P176 
Cotrupi S, Ranzani D, Maier JAM (2005) Impact of modeled microgravity on microvascular endothelial cells. Biochimica et Biophysica Acta (BBA) - Molecular Cell Research 1746(2): 163-168

Dai K, Wang Y, Yan R, Shi Q, Wang Z, Yuan Y, Cheng H, Li S, Fan Y, Zhuang F (2009) Effects of microgravity and hypergravity on platelet functions. Thrombosis and Haemostasis 101(5): 902-910

Deakin AM, Payne AN, Whittle BJ, Moncada S (1995) The modulation of IL-6 and TNFalpha release by nitric oxide following stimulation of J774 cells with LPS and IFNgamma. Cytokine 7(5): 408-416

Douville EC, Kempczinski RF, Birinyi LK, Ramalanjaona GR (1987) Impact of endothelial cell seeding on long-term patency and subendothelial proliferation in a smallcaliber highly porous polytetrafluoroethylene graft. Journal of Vascular Surgery 5(4): 544550

Gamboa A, Okamoto LE, Raj SR, Diedrich A, Shibao CA, Robertson D, Biaggioni I (2013) Nitric oxide and regulation of heart rate in patients with postural tachycardia syndrome and healthy subjects. Hypertension 61(2): 376-381

Gimbrone MA, Lelkes P (2003) Mechanical Forces and the Endothelium, The Netherlands: Hardwood Academic Publishers

Grenon SM, Jeanne M, Aguado-Zuniga J, Conte MS, Hughes-Fulford M (2013) Effects of gravitational mechanical unloading in endothelial cells: association between caveolins, inflammation, and adhesion molecules. Scientific Reports 3: 1494

Grimm D, Wehland M, Pietsch J, Aleshcheva G, Wise $\mathrm{P}$, van Loon J, Ulbrich C, Magnusson NE, Infanger M, Bauer J (2014) Growing tissues in real and simulated microgravity: new methods for tissue engineering. Tissue Engineering Part B, Reviews 20(6): 555-566

Hammond TG, Hammond JM (2001) Optimized suspension culture: the rotating-wall vessel. American Journal of Physiology - Renal Physiology 281(1): F12-F25
Hristov M, Erl W, Weber PC (2003) Endothelial progenitor cells: mobilization, differentiation, and homing. Arteriosclerosis, Thrombosis, and Vascular Biology 23: 11851189

Hughes-Fulford M (2011). To infinity ... and beyond! Human spaceflight and life science. Federation of American Societies for Experimental Biology (FASEB) Journal 25(9): 2858-2864

Jacquier-Sarlin MR, Fuller K, Dinh-Xuan AT, Richard M-J, Polla BS (1994) Protective effects of hsp70 in inflammation. Experientia 50(11): 1031-1038

Kiang J (1998) Heat shock protein 70 kDa: molecular biology, biochemistry, and physiology. Pharmacology \& Therapeutics 80(2): 183-201

Klaus DM (2001) Clinostats and bioreactors. Gravitational and Space Biology Bulletin 14(2): 55-64

Kunz GA, Liang G, Cuculi F, Gregg D, Vata KC, Shaw LK, Goldschmidt-Clermont PJ, Dong C, Taylor DA, Peterson ED (2006) Circulating endothelial progenitor cells predict coronary artery disease severity. American Heart Journal 152(1): 190-195

Levin EG, Marzec U, Anderson J, Harker LA (1984) Thrombin stimulates tissue plasminogen activator release from cultured human endothelial cells. Journal of Clinical Investigation 74(6): 1988-1995

Luo X, Zuo X, Zhou Y, Zhang B, Shi Y, Liu M, Wang K, McMillian DR, Xiao X (2008) Extracellular heat shock protein 70 inhibits tumour necrosis factor-a induced proinflammatory mediator production in fibroblast-like synoviocytes. Arthritis Research \& Therapy 10: R41

Maiello M, Boeri D, Podesta F, Cagliero E, Vichi M, Odetti P, Adezati L, Lorenzi M (1992) Increased expression of tissue plasminogen activator and its inhibitor and reduced fibrinolytic potential of human endothelial cells cultured in elevated glucose. Diabetes 41(8): 1009-1015

Maier JAM, Cialdai F, Monici M, Morbidelli L (2015) The impact of microgravity and hypergravity on endothelial cells. BioMed Research International Volume 2015: 434803 
Martinez EM, Yoshida MC, Candelario TLT, Hughes-Fulford M (2015) Spaceflight and simulated microgravity cause a significant reduction of key gene expression in early Tcell activation. American Journal of Physiology: Regulatory, Integrative, and Comparative Physiology 308(6): R480-R488

May LT, Torcia G, Cozzolino F, Ray A, Tatter SB, Santhanam U, Sehgal PB, Stern D (1989) Interleukin-6 gene expression in human endothelial cells: RNA start sites, multiple IL-6 proteins, and inhibition of proliferation. Biochemical and Biophysical Research Communications 159(3): 991-998

Motlagh D, Allen J, Hoshi R, Yang J, Lui K, Ameer G (2007) Hemocompatibility evaluation of poly(diol citrate) in vitro for vascular tissue engineering. Journal of Biomedical Materials Research, Part A 82(4): 907-916

Mukai CN, Lathers CM, Charles JB, Bennett BS (1994) Cardiovascular responses to repetitive exposure to hyper- and hypogravity states produced by parabolic flight. Journal of Clinical Pharmacology 34(5): 472-479

Murasawa S, Asahara T (2005) Endothelial progenitor cells for vasculogenesis. Physiology 20: 36-42

Orshal JM, Khalil RA (2004) Reduced endothelial NO-cGMP-mediated vascular relaxation and hypertension in IL-6-infused pregnant rats. Hypertension 43(2): 434-444

Piro M, Giubilato G, Pinnelli M, Giordano Sciacca P, Biasucci LM (2005) Endothelium and inflammation. Panminerva Medica 47(2): $75-80$

Radtke AL, Herbst-Kralovetz MM (2012) Culturing and applications of rotating wall vessel bioreactor derived 3D epithelial cell models. Journal of Visualized Experiments 62: 3868

Ramaswamy V, Dirr EW, Allen JB (2015) The effect of simulated microgravity on differentiation of porcine blood-derived vascular stem cells. Stem Cells and Development 25(4): 329-336

Ravagnan L, Gurbuxani S, Susin SA, Maisse C, Daugas E, Zamzami N, Mak T, Jäättelä M, Penninger JM, Garrido C, Kroemer G (2001) Heat-shock protein 70 antagonizes apoptosis- inducing factor. Nature Cell Biology 3(9): 839-843

Ritz BW, Lelkes PI, Gardner EM (2006) Functional recovery of peripheral blood mononuclear cells in modeled microgravity. Federation of American Societies for Experimental Biology (FASEB) Journal 20(2): 305-307

Rubanyi GM (1993) The role of endothelium in cardiovascular homeostasis and diseases. Journal of Cardiovascular Pharmacology 22(Suppl 4): S1-S14

Spisni E, Bianco MC, Griffoni C, Toni M, D'Angelo R, Santi S, Riccio M, Tomasi V (2003) Mechanosensing role of caveolae and caveolar constituents in human endothelial cells. Journal of Cellular Physiology 197(2): 198-204

Spisni E, Toni M, Strillacci A, Galleri G, Santi S, Griffoni C, Tomasi V (2006) Caveolae and caveolae constituents in mechanosensing: effect of modeled microgravity on cultured human endothelial cells. Cell Biochemistry and Biophysics 46(2): 155-164

Stern DM, Esposito C, Gerlach H, Gerlach M, Ryan J, Handley D, Nawroth P (1991) Endothelium and regulation of coagulation. Diabetes Care 14(2): 160-166

Trepels T, Zeiher AM, Fichtlscherer S (2009) The endothelium and inflammation. Endothelium: Journal of Endothelial Cell Research 13(6): 423-429

Unsworth BR, Lelkes PI (1998) Growing tissues in microgravity. Nature Medicine 4(8): 901907

Urbich C, Dimmeler S (2004) Endothelial progenitor cells: characterization and role in vascular biology. Circulation Research 95(4): 343-353

Uva BM, Masini MA, Sturla M, Prato P, Passalacqua M, Giuliani M, Tagliafierro G, Strollo F (2002) Clinorotation-induced weightlessness influences the cytoskeleton of glial cells in culture. Brain Research 934(2): 132-139

Van Guilder GP, Hoetzer GL, Smith DT, Irmiger HM, Greiner JJ, Stauffer BL, DeSouza CA (2005) Endothelial t-PA release is impaired in overweight and obese adults but can be improved with regular aerobic exercise. American Journal of Physiology, 


\section{Ramaswamy et al. -- Simulated Microgravity Alters Blood-Derived Endothelial Cell Functions}

Endocrinology, and Metabolism 289(5): E807-E813

van Hinsbergh VWM (2012) Endothelium - role in regulation of coagulation and inflammation. Seminars in Immunopathology 34(1): 93-106

Vaziri ND, Ding Y, Sangha DS, Purdy RE (2000) Upregulation of NOS by simulated microgravity, potential cause of orthostatic intolerance. Journal of Applied Physiology 89(1): 338-344

Versari S, Longinotti G, Barenghi L, Maier JAM, Bradamante S (2013) The challenging environment on board the International Space Station affects endothelial cell function by triggering oxidative stress through thioredoxin interacting protein overexpression: the ESA-SPHINX experiment. Federation of American Societies for Experimental Biology (FASEB) Journal 27(11): 4466-4475

Versari S, Villa A, Bradamante S, Maier JAM (2007) Alterations of the actin cytoskeleton and increased nitric oxide synthesis are common features in human primary endothelial cell response to changes in gravity. Biochimica et Biophysica Acta 1773(11): 1645-1652

Villa A, Versari S, Maier JAM, Bradamante S (2005) Cell behavior in simulated microgravity: a comparison of results obtained with RWV and RPM. Gravitational and Space Biology Bulletin 18(2): 89-90

Wehland M, Ma X, Braun M, Hauslage J, Hemmersbach R, Bauer J, Grosse J, Infanger M, Grimm D (2013) The impact of altered gravity and vibration on endothelial cells during a parabolic flight. Cellular Physiology and Biochemistry: International Journal of Experimental Cellular Physiology, Biochemistry, and Pharmacology 31(2-3): 432451

Yamamoto K, Takahashi T, Asahara T, Ohura N, Sokabe T, Kamiya A, Ando J (2003) Proliferation, differentiation, and tube formation by endothelial progenitor cells in response to shear stress. Journal of Applied Physiology 95(5): 2081-2088

Yates BJ, Kerman IA (1998) Post-spaceflight orthostatic intolerance: possible relationship to microgravity-induced plasticity in the vestibular system. Brain Research Reviews 28(1-2): 73-82

Zampetaki A, Kirton JP, Xu Q (2008) Vascular repair by endothelial progenitor cells. Cardiovascular Research 78(3): 413-421

Zhang Y, Sang C, Paulsen K, Arenz A, Zhao Z, Jia X, Ullrich O, Zhuang F (2010) ICAM-1 expression and organization in human endothelial cells is sensitive to gravity. Acta Astronautica 67(9): 1073-1080 